\title{
O Ensino-aprendizagem da Relação Médico- paciente: Estudo de Caso com Esudantes do Último Semestre do Curso de Medicina*
}

\author{
Teaching and Learning the Doctor-patient \\ Relationship: Case-study with Medical \\ Students in the Last Semester
}

Suely Grosseman ${ }^{I}$ Carolina Stoll ${ }^{I I}$

\section{PALAVRAS-CHAVE \\ - Relações Médico-Paciente; \\ - Estudantes de Medicina; \\ - Aprendizagem; \\ - Assistência Centrada no Paciente; \\ - Ética; \\ - Educação Médica.}

\section{KEY WORDS}

- Doctor-Patient Relationship;

- Students, Medical;

- Learning;

- Patient-centered Care;

- Ethics;

- Medical Education. 


\section{INTRODUÇÃO}

A relação médico-paciente é um processo especial de interação humana, que é a base da prática clínica em suas dimensões técnica, humanística, ética e estética ${ }^{1,2,3}$. Como qualquer processo de interação interpessoal, essa relação é mediada pela comunicação ${ }^{4}$.

Entre seus benefícios estão: maior precisão na identificação dos problemas do paciente, com promoção do raciocínio clínico; maior adesão ao tratamento; melhor entendimento pelos pacientes de seus problemas, das investigações conduzidas e das opções de tratamento; menor incidência de queixas de erro médico; e maior satisfação para médico e paciente ${ }^{5-8}$.

Pesquisas realizadas desde a década de 1970 têm constatado associação entre comunicação efetiva e influência positiva na saúde física e emocional do paciente ${ }^{9-11}$. Mas a demonstração de problemas na área da comunicação em saúde tem sido freqüente, como a percepção de pacientes que suas preocupações não são elucidadas pelos médicos, o não diagnóstico de problemas psicossociais e psiquiátricos na prática médica, a falta de aconselhamento em saúde pelo médico ao paciente e o não entendimento ou a não lembrança pelos pacientes sobre o que o médico diz sobre o diagnóstico e/ou tratamento ${ }^{12-15}$.

As habilidades de comunicação podem ser ensinadas ${ }^{16-18}$. Em 1995, foi adotada uma resolução pelo Comitê de Acreditação em Educação Médica dos EUA (Liaison Committee on Medical Education) e pelo Comitê de Acreditação das Escolas Médicas Canadenses (Committee on Acreditation of Canadian Medical Schools) segundo a qual "as habilidades de comunicação são fundamentais para a educação e o funcionamento eficiente dos médicos" e que "deve haver instrução e avaliação específicas destas habilidades, já que estão relacionadas a responsabilidades médicas, incluindo comunicação com pacientes, famílias, colegas e outros profissionais de saúde"19.

As escolas médicas têm não só a oportunidade, mas a responsabilidade de ensinar e avaliar as habilidades de comunicação ${ }^{6}$, e estudos demonstram sua promoção após intervenções educacionais ${ }^{16-18,20,21}$

No Brasil, as diretrizes curriculares para o curso de graduação em Medicina, homologadas em 2001, contemplam a importância das habilidades de comunicação ${ }^{22}$, e a Comissão Nacional de Residência Médica, em 2004, deliberou que os concursos para admissão de residentes deveriam incluir uma segunda fase, constituída de prova prática, já que "a avaliação das habilidades e comportamentos constitui elemento essencial à seleção do candidato" ${ }^{23}$.

Tendo em vista a importância da relação médico-paciente na prática médica e a possibilidade de seu ensino-apren- dizagem, o objetivo deste estudo foi conhecer a percepção de estudantes da última fase do curso de Medicina de uma universidade federal do Sul do Brasil sobre seu processo de ensino-aprendizagem da relação médico-paciente.

\section{MÉTODO}

Este estudo é parte de uma pesquisa mais abrangente ${ }^{24}$ desenvolvida por abordagem mista (qualitativa e quantitativa), usando-se o estudo de caso.

Os sujeitos foram 25 estudantes, selecionados de forma aleatória, por sorteio, do universo de 47 estudantes pertencentes ao décimo segundo semestre do curso de graduação em Medicina de uma universidade federal do Sul do Brasil.

Após submissão e aprovação do projeto pelo Comitê de Ética em Pesquisa com Seres Humanos, sob o protocolo no 309/04, foi realizado projeto piloto com um estudante (selecionado por sorteio) e verificou-se que o método não necessitava alterações.

Após esta etapa, os acadêmicos sorteados foram convidados a participar do estudo, sendo explicado o teor da pesquisa, assegurando-se sigilo e anonimato. Após aceitação, foi solicitada a assinatura do Termo de Consentimento Livre e Esclarecido. Os dados foram coletados em 2005, por meio de entrevista semi-estruturada em profundidade, guiada por questões norteadoras. Na pesquisa que deu origem a este estudo, havia outras questões relativas à percepção e à atitude dos estudantes quanto à relação médico-paciente, que não serão abordadas neste artigo.

A análise dos dados foi realizada pelo método de AnáliseReflexão-Síntese, preconizado por Patrício, que pressupõe o movimento de análise de conteúdo e a síntese mediados pela sensibilidade e pela razão $0^{25}$. A abordagem quantitativa foi usada para descrever a distribuição de freqüência, sem intenção de realizar comparações.

\section{A PERCEPÇÃO DOS ENTREVISTADOS SOBRE O APRENDIZADO DA RELAÇÃO MÉDICO-PACIENTE}

Em relação à forma como é aprendida a relação médico-paciente, 21 estudantes (84\%) mencionaram o aprendizado com modelos, observando o comportamento de médicos, professores, residentes, colegas e outros profissionais da saúde. Nesta reflexão, o aprendizado aparece como o que fazer [modelos considerados adequados, mencionados por 20 estudantes $(80 \%)$ ] e o que não fazer [modelos considerados não adequados, citados por $15(60 \%)]$ :

Acho que através de exemplos... alguns que eu olhava, achava legal copiar, outros que eu olhava, achava horrível e deixava de lado... Como é difícil ver 
algum médico que dê um bom exemplo de relação médico-paciente, a gente fica meio perdido, se sente inseguro, tem muito medo... E ninguém diz pra gente como seria o correto, ninguém fala nada! Se tu não pára pra pensar, acha que aquilo é o normal e segue fazendo igual.

Eu vejo assim: 'Meu Deus, isso eu não quero fazer'. Então, a gente aprende tanto com os bons exemplos como com os maus exemplos. No caso, tu tens que ter discernimento. Porque tem aqueles que olham os maus exemplos e acham o máximo.

Dezessete estudantes (68\%) citaram o aprendizado na prática, em atendimentos realizados no dia-a-dia [14 (56\%)], estágios extracurriculares [9 (36\%)] e no internato [3 (12\%)].

Eu acho que os estágios contribuíram muito pra mim, porque eu não cheguei no internato tão crua [...] me ajudou a entrar em contato com o paciente, ele ser teu paciente, tem isso de tu seres o responsável, tu teres que colher a história dele de alguma forma... Dá pra desenvolver bem isso no internato, talvez até melhor do que nos estágios que eu fiz, que no geral eram estágios de emergência e que não valorizam muito isso.

Doze $(48 \%)$ referiram que atividades teóricas, como aulas [9 $(36 \%)$ ou leitura de livros e artigos [6 (24\%)] também são uma boa forma de aprender a relação médico-paciente. Mas 4 entrevistados (16\%) mencionaram não acreditar que isto seja possível:

... isso ninguém ensina, é uma coisa que a gente pega na prática... Quanto mais paciente você vê, mais aprende... Acho que a gente aprende no dia-a-dia. Não tem um professor que vai ensinar relação médico-paciente. Aprendi na emergência, nas enfermarias, aprendi errando também. Já briguei com pacientes... Eu acho que isso não é teórico, é prático.

Outro fatores aludidos como influentes no aprendizado foram os relacionamentos interpessoais [11 (44\%)], a opinião dos pacientes [7 $(28 \%)$ ] e a vivência como paciente [5 $(20 \%)]$.

... nossos relacionamentos [...]. Eu sempre dizia para todos quando eu era monitor: 'Vai fazer festa, vai conhecer gente'. Eu acho que isso é uma maneira de a gente vivenciar relação... se a gente está só voltada para os livros [...]... não vai ter feeling na hora de conversar com alguém.
Para nove entrevistados (36\%), a relação médico-paciente é algo individual, "é uma coisa que... já tem embutido", "depende de pessoa para pessoa... umas têm uma relação médico-paciente inerente; outras, por mais que tenham contato, fiquem anos dentro do hospital, nunca vão ter uma relação perfeita. Porque é inerente da pessoa o fato de se comunicar bem, ter uma empatia melhor com o paciente".

A importância da relação professor-aluno foi mencionada por três entrevistados $(12 \%)$ :

...o primeiro contato que a gente tem com a relação médico-paciente na faculdade de Medicina é a relação professor-aluno. A maneira como a gente vê o professor se preocupando em ensinar o aluno, em saber o nome e reconhecer aluno por aluno, dedicar tempo ensinando, é o primeiro grande contato em que a gente pode se espelhar um pouco. A maneira como o professor trata a gente é a maneira como eu muitas vezes quis tratar meus pacientes depois... Acho que tem uma relação muito semelhante, de professor-aluno e médico-paciente... Não só acho, como pude comprovar na prática, muitas vezes, a mesma maneira como o professor tratava a gente, tratava os pacientes...

Cinco estudantes (20\%) reportaram a importância de refletir sobre seu relacionamento com os pacientes, como forma de aperfeiçoá-lo.

Em relação às aulas sobre relação médico-paciente durante o curso, uma entrevistada ( $4 \%$ ) considerou bom e suficiente esse ensino no curso: "Eu acho que eles ensinam bem. Na psicologia, na psiquiatria, durante as aulas práticas... Eu acho que a relação médico-paciente está sendo bem abordada durante o curso". Mas 9 (36\%) julgaram que podia ter sido melhor, e 2 $(8 \%)$ acharam que simplesmente não ocorreu.

Entre os nove entrevistados que mencionaram as aulas teóricas (36\%), para alguns estas poderiam ser aprofundadas e estão "um pouco aquém da real necessidade... uma aula sobre relação médico-paciente em psiquiatria, uma aula em introdução ao exame clínico, uma aula na pediatria preventiva e social, uma aula em psicologia médica também, mas ainda é muito escasso".

Foi lembrado o grande aprendizado obtido em aulas práticas $[8(32 \%)]$ :

De todas as aulas práticas que eu já tive, essa foi a que mais me marcou. A gente teve um debate antes da aula sobre relação médico-paciente, então nós fomos fazer a consulta. Era um paciente idoso, devia ter uns 
80 anos, muito sisudo, muito sério, falava não olhando pra gente, falava brabo, monossilábico, às vezes, porque não estava com a menor paciência pra responder aquilo tudo de novo. E a doutora começou a conduzir a anamnese, a fazer aquelas perguntas clássicas: nome, idade... Quando chegou em procedência, ela disse: 'É uma cidade que tem plantações lindas!'. Aí ele levantou a cabeça. 'O senhor planta?', disse a doutora. 'Eu planto. Planto isso, planto aquilo'. 'E como é lá?'. 'Ah, eu acordo cedinho, professora, pego meu trator, minhas sementinhas, dou comida pros meus bichinhos...'. Começou a falar, a sorrir e a brincar, e todo mundo ficou envolvido naquela situação. Todo mundo percebeu o quanto o cara tinha mudado... Quando ele terminou o relato, começou a chorar de maneira bem tímida, $e$ a doutora foi lá, tocou no ombro dele: 'O senhor está com saudade, mas vai voltar logo pra lá. Continuou a anamnese, e o cara se entregou completamente a ela, em termos de conversar, de ter paciência pra gente fazer perguntas, pra gente examinar [...]. Ela mostrou claramente que você leva três minutos pra escutar o cara e mudou completamente a conduta da anamnese. Eu acho que todo aluno devia ter visto uma anamnese daquela.

Mas cinco (20\%) consideraram ter aprendido pouco ou "desaprendido" a relação nessas aulas:

[...] na parte clínica... aquela coisa de entrar os 12 num quartinho, pedir pro paciente se despir, mostrar alguma coisa e sair correndo [...]. Ninguém se preocupava se ele tava com bom humor, se já tinha contado aquela história 2.500 vezes, se tinha alguma queixa [...]. E o próprio fato de botar 15 pessoas dentro do quarto para ver o cara, pelo amor de Deus, o que é isso?

Quinze entrevistados gostariam de ter aprendido melhor a relação médico-paciente. Suas sugestões para melhorar o ensino incluíram maior ênfase no tema ao longo de todo o curso de Medicina, com reforço do ensino teórico durante o período de vivência prática $(87 \%)$, treinamento para abordar o paciente em situações específicas ( $27 \%$ ) e a criação de um módulo ou disciplina para abordar o tema (20\%).

... discussão de casos éticos, como abordar um paciente com câncer, uma gestante que perdeu o neném, uma paciente que soube que é HIV positiva... Trazer um oncologista pra falar, trazer um obstetra pra falar, pelo menos saber conduzir certas situações. Porque eu tenho certeza de que muitos dos meus colegas e eu, inclusive, quando saírem da universidade, vão se deparar com uma situação com que não vão saber lidar. E aí? Quando a situação bater na tua cara... Eu nunca me perguntei como vou lidar com uma mãe que chegar com um ultra-som mostrando anencefalia. O que é que eu vou dizer para esta mulher?

Ética que a gente tem no básico fica muito distante da prática... Eu acho que precisava reforçar mais para o final do curso. Muitas coisas que a gente achava absurdas quando estava na primeira, segunda fase, quando a gente estudou ética, hoje em dia a gente vê nossos colegas fazendo e acha que aquilo é normal. E, às vezes, a gente precisa parar para pensar na ética, na relação médico-paciente, para não acabar ficando igual a essas pessoas.

Foram também sugeridos maior valorização das disciplinas relacionadas à interação médico-paciente, atividades práticas sob orientação dos professores e contato precoce com a comunidade e os pacientes: "Na medicina, o primeiro contato que a gente tem é com cadáver... Então, a gente começa a ver pelo lado avesso. A gente vê cadáver, depois vê tecido, célula... Talvez fosse mais interessante começar pelo ser humano inteiro e depois desmontar em órgãos, tecidos, células...".

A maior valorização da relação médico-paciente por meio de sua avaliação durante o curso ou em provas de residência e o preparo dos professores para abordar o seu ensino também foram aludidos [por $3(12 \%)$ ].

A oportunidade de refletir sobre o tema ao participar do estudo foi mencionada: “Acho que é uma idéia bem legal, até pra quem está fazendo a entrevista, parar para pensar nessas coisas que a gente não tem... o que acham importante na relação médico-paciente. Não lembro de ter parado para pensar nisso".

\section{A RELAÇÃO MÉDICO-PACIENTE: DA TEORIA À PRÁTICA E DA PRÁTICA À TEORIA}

Os modelos, a prática extracurricular e o internato foram considerados os principais recursos de aprendizado da relação médico-paciente pelos estudantes, tendo sido julgadas escassas as aulas teóricas e pouco aproveitadas as oportunidades de aprendizagem nas práticas. As disciplinas mencionadas em que se abordava a relação médico-paciente foram psicologia médica, psiquiatria, semiologia e pediatria preventiva e social. 
Esses resultados são similares aos encontrados por Batista e Rossi num grupo de iniciantes da residência em clínica médica num hospital geral de São Paulo ${ }^{26}$. Eles ressaltam que o currículo oculto - normas e valores efetivamente transmitidos pelas escolas, presentes em todos os momentos de aprendizagem, mas não de forma explícita — ainda é uma das principais estratégias do aprendizado da relação.

Apesar de os estudantes acreditarem que a aquisição de habilidades de comunicação para o exercício da medicina seja mais uma questão de prática, que pode ser adquirida por conta própria, a experiência, por si só, não garante esta aprendizagem. Isso pode levar à repetição continuada de erros, diminuição de empatia do estudante e deterioração na relação médico-paciente 16,27-30 $^{\text {. }}$

A maioria dos estudantes entrevistados gostaria que a relação médico-paciente fosse mais abordada durante o curso de Medicina, em momentos específicos ou ao longo de todo o curso, durante as aulas gerais ou em disciplinas próprias, pela abordagem de situações especiais, havendo a percepção da necessidade de treinar os professores para o seu ensino. Alguns sugeriram que o tema permeasse todo o curso de Medicina.

Inconsistências entre o que se aprende na sala de aula e o que se vê na prática, com mensagens divergentes, podem levar os estudantes a desvalorizar a relação médico-paciente e a concluir que as habilidades de comunicação não são relevantes no seu cuidado ${ }^{6,29}$.

Uma situação comum de mensagens divergentes é a orientação para conduzir a anamnese com perguntas mais direcionadas pelos professores de clínica e a orientação para conduzi-la com pergunta abertas, sendo mais reflexivos. Por isso, é importante aperfeiçoar os professores no desenvolvimento de um currículo para a comunicação que possibilite a revisão, discussão e prática dos métodos correntes de ensino e avaliação das habilidades de comunicação ${ }^{29}$. Por outro lado, é fundamental que os professores da comunicação criem experiências educacionais relevantes para a prática clínica ${ }^{6}$.

As habilidades de comunicação vêm sendo ensinadas em várias escolas médicas, de diversas formas. Yedidia et $a l^{17}$, investigando alunos do terceiro ano de três escolas médicas americanas, foram expostos a um novo currículo, que visava ensinar um grupo comum de habilidades de comunicação. Embora estas habilidades tenham melhorado durante o terceiro ano do curso, tanto no grupo-controle quanto no da intervenção, a melhora foi significativamente maior nos estudantes expostos à intervenção.

Uma universidade da Filadélfia implementou um currículo para ajudar os estudantes a melhorar várias habilidades clínicas durante seu estágio em medicina interna no terceiro $a^{13}$. A cada período de quatro semanas, os estudantes escolhiam uma competência a ser trabalhada, revisavam o módulo sobre esta competência no website do estágio e realizavam uma revisão de literatura ${ }^{16}$. A revisão da literatura e a oportunidade de observar o preceptor desempenhando as competências foram os componentes do currículo mais freqüentemente apontados como úteis ${ }^{13}$.

Uma pesquisa realizada em 1997 e 1998 determinou o status do ensino e da avaliação das habilidades de comunicação em escolas médicas nos Estados Unidos, Canadá e Porto Rico 6 . De 89 escolas que responderam questões sobre os métodos de ensino utilizados, os citados, em ordem de freqüência, foram: combinação de discussão, observação e prática, discussões em pequenos grupos e seminários, apresentações orais, entrevista de pacientes simulados pelos alunos, observação dos professores com pacientes reais e entrevistas com pacientes verdadeiros pelos estudantes. Metade das escolas (44,9\%) utiliza visitas (rounds) para ensinar habilidades de comunicação. Aproximadamente $40 \%$ das escolas informaram utilizar gravações em vídeo das interações dos estudantes, que, segundo Makoul ${ }^{6}$, são um material importante para reflexão e auto-avaliação. Todas as escolas informaram fazer avaliação dos estudantes por meio de feedback pelos professores durante as sessões de ensino. Métodos de avaliação mais objetivos, como os que envolvem pacientes simulados, são menos utilizados.

Uma série de recomendações para desenvolver e implementar programas de ensino e avaliação da comunicação foi gerada durante a Conferência Internacional sobre o Ensino da Comunicação em Medicina ${ }^{31}$.

Foi criado o projeto Ensino Médico na Graduação para o século 21 (Undergraduate Medical Education for the $21^{\text {st }}$ Century - UME-21) ${ }^{32}$, para implementar e avaliar currículos inovadores para escolas de Medicina que oferecessem conhecimentos, habilidades e atitudes necessárias a um cuidado de alta qualidade aos pacientes em meio ao sistema de saúde do século 21. De 1997 a 2002, 18 escolas participantes do projeto deveriam incluir inovações e objetivos de ensino em nove áreas, dentre as quais desenvolvimento de habilidades de comunicação e de relações eficientes entre cuidador e paciente. Todas as escolas participantes do projeto tinham o ensino de habilidades de comunicação incluído no currículo. Doze desenvolveram programas para melhorar as habilidades comunicacionais dos estudantes do terceiro e quarto anos. Algumas reforçaram habilidades básicas de comunicação nos estágios clínicos. Outras proporcionaram oportunidades para a prática de habilidades avançadas, como lidar com assuntos delicados ${ }^{29}$ 
Vários métodos foram utilizados para alcançar os objetivos de ensino. A maioria incluiu uma combinação de leituras, palestras, discussões e atividades em pequenos grupos (representação de papéis com pacientes simulados, interação com pacientes simulados, análise de consultas gravadas em fitas de som ou vídeo). Outros métodos utilizados foram: observar o preceptor ou uma enfermeira conversar com um paciente sobre a doença crônica deste; discutir com preceptores sobre como lidar com situações de conflito com o paciente; escrever sobre seus pacientes, incluindo informações sobre as preocupações destes, o objetivo da visita e nível de estresse emocional; visitar pacientes portadores de doenças crônicas, realizar uma apresentação numa escola sobre a interrupção do tabagismo e tentar modificar um de seus comportamentos prejudiciais (mal adaptive behaviors) ${ }^{29}$.

Os pacientes simulados (standardized patients) e a simulação de consultas médicas por meio de dramatização permitiram que os estudantes observassem e discutissem seu próprio desempenho e o de colegas. Várias escolas participantes iniciaram novos métodos para avaliar as habilidades de comunicação de seus estudantes. Estes incluíram exames clínicos objetivos estruturados com pacientes simulados (Objective Structured Clinical Examination - Osce), bem como feedback e perguntas baseados em gravações de consultas realizadas pelos estudantes ${ }^{29}$.

$\mathrm{Na}$ Ghent University (Bélgica), o novo currículo médico, elaborado em 1999, implementou o treinamento teórico e prático em comunicação em todos os anos do curso. Entre os métodos didáticos utilizados estão a demonstração das habilidades em vídeos e leitura de casos de histórias de pacientes, dramatização com colegas ou pacientes simulados em pequenos grupos (de 5 a 10 estudantes) com foco na filmagem das consultas reais, que proporcionam uma idéia do desempenho de cada estudante. A cada ano, os estudantes são avaliados pelo Osce, e aqueles com baixo desempenho recebem treinamento extra ${ }^{33}$.

Foi sugerido o contato precoce com a comunidade. Com a adesão às novas diretrizes curriculares, vários cursos de Medicina têm inserido os estudantes desde a primeira fase do curso na comunidade. Um estudo recente na Faculdade de Marília demonstrou o valor desse contato no crescimento pessoal e profissional percebidos por estudantes das primeiras fases do curso de Medicina ${ }^{34}$.

Apesar de alguns entrevistados terem considerado que a relação médico-paciente só pode ser aprendida na prática, além dos artigos citados neste estudo, existem diversos textos de valor inestimável para seu aprendizado, sendo aqui apontados alguns $\mathrm{s}^{35-39}$.
Na opinião de Haq, um desafio para o futuro é treinar os professores a servir de modelo em habilidades de comunicação efetivas, observar as interações dos estudantes com os pacientes e fornecer feedback confiável, criando um ambiente que demonstre e reforce consistentemente a importância da comunicação médico-paciente e que ajude os estudantes a melhorar suas habilidades ${ }^{29}$. Professores que entendem o valor de uma comunicação efetiva - o que nem sempre aumenta significativamente o tempo de atendimento - são mais propensos a demonstrar e a reforçar estas habilidades ${ }^{28,35}$.

Deve-se ressaltar que recursos humanos e financeiros são necessários para treinar os professores a desenvolverem um currículo longitudinal, compreensivo, para ensinar e avaliar as competências dos estudantes sistematicamente ${ }^{36}$.

Além do ensino das estratégias de comunicação para o aprimoramento da relação médico-paciente, trabalhar na área da medicina pode ser árduo, estressante e até mesmo prejudicial à saúde do médico. Os estudantes devem ter a oportunidade de aprender a desenvolver o autoconhecimento, além de estratégias para lidar com o estresse. Com planejamento adequado, este tipo de atividade pode acontecer em pequenos grupos de discussão. Além disso, métodos usados para o feedback devem encorajar os estudantes a refletir sobre seus pontos fortes e a identificar áreas em que podem melhorar. $\mathrm{O}$ ensino e a avaliação que respondem às necessidades dos que aprendem tendem a promover o cuidado médico que responde às necessidades dos pacientes ${ }^{31}$.

\section{CONSIDERAÇÕES FINAIS}

Ao longo da pesquisa, percebeu-se que a grande maioria se esforça para aperfeiçoar a relação médico-paciente, procurando aprender com os modelos e melhorar no dia-a-dia. E também o quanto gostaria que a relação médico-paciente fosse mais abordada durante o curso, por meio de maior ênfase, de aulas ou disciplinas sobre o tema ou da abordagem de situações específicas. A maioria considerou ter pouca orientação sobre as habilidades comunicacionais consideradas fundamentais ao bom exercício da medicina, e muitos desconhecem os diversos métodos pelos quais estas podem ser ensinadas.

Esta é uma oportunidade que não pode ser perdida: estudantes interessados sobre o tema e motivados a aprender. Sabese que muitas universidades no Brasil estão dando ênfase ao aspecto atitudinal, no qual se inclui a relação médico-paciente.

O novo currículo do curso de Medicina da faculdade em estudo está no décimo semestre e já insere os estudantes na comunidade desde a primeira fase do curso e aborda um pouco mais de conteúdo relacionado a este tema. Espera-se que a relação médico-paciente continue sendo enfatizada até o último ano do curso. 
Tendo como perspectiva fornecer subsídios para aprimorar o ensino-aprendizagem da relação médico-paciente durante o curso de Medicina, sugere-se: a criação de espaços para reflexão, mediados por professores ou médicos preparados, em que estudantes possam discutir seu aprendizado, seus relacionamentos e suas angústias; maior preparo dos professores, que são indispensáveis no ensino das habilidades de comunicação, no feedba$c k$ fornecido aos estudantes e na demonstração do ideal a seguir; desenvolver mais pesquisas para aprofundar os conhecimentos sobre o tema; estimular o desenvolvimento de pesquisas com abordagem qualitativa ao longo do curso para aprimorar as habilidades de comunicação, em especial a capacidade de ouvir, importante em qualquer relacionamento, inclusive na relação médico-paciente.

\section{REFERENCIAS}

1. Teutsch C. Patient-doctor communication. Med Clin North Am 2003; 87(5):1115-45.

2. Grosseman S, Patrício ZM. A relação médico-paciente e o cuidado humano: subsídios para promoção da educação médica. Rev Bras Educ Méd. 2004; 28(2):99-105.

3. Bascunan RM. Changes in physician-patient relationship and medical satisfaction. Rev Med Chil 2005;133(1):11-6.

4. Coyle N, Sculco L. Communication and the patient/physician relationship: a phenomenological inquiry. J Support Oncol 2003;1(3):206-15

5. Maguire P, Pitceathly C. Key communication skills and how to acquire them. BMJ 2002; 325(7366):697-700.

6. Association of American Medical Colleges. Contemporary issues in medicine. Report III of medical school objectives project. Washington (DC): Association of American Medical Colleges;1999.

7. Levinson W. Physician-patient communication. A key to malpractice prevention. JAMA. 1994; 272(20):1619-20.

8. Stewart M, Meredith L, Brown JB, Galajda J. The influence of older patient-physician communication on health and health-related outcomes. Clin Geriatr Med 2000; 16(1):2536, vii-viii.

9. Stewart M A. Effective physician-patient communication and health outcomes: a review. Cmaj 1995;152(9):1423-33.

10. Heszen-Klemens I, Lapinska E. Doctor-patient interaction, patients' health behavior and effects of treatment. Soc Sci Med 1984;19(1):9-18.

11. Orth JE, Stiles WB, Scherwitz L. Interviews and hypertensive patients' blood pressure control. Health Psychology $1987 ; 6: 29-42$.
12. Stewart MA, McWhinney IR, Buck CW. The doctor/patient relationship and its effect upon outcome. J R Coll Gen Pract 1979; 29(199):77-81.

13. Schulberg HC, Burns BJ. Mental disorders in primary care: epidemiologic, diagnostic, and treatment research directions. Gen Hosp Psychiatry 1988; 10(2):79-87.

14. Ley P. Communication with patients: improving satisfaction and compliance. London: Croom-Helm;1988.

15. Steine S, Finset A, Laerum E. A new, brief questionnaire (PEQ) developed in primary health care for measuring patients' experience of interaction, emotion and consultation outcome. Fam Pract 2001; 18 (4):410-8.

16. Brody DS, Ryan K, Kuzma MA. Promoting the development of doctoring competencies in clinical settings. Fam Med 2004;36 (Supl.S): 105-9.

17. Yedidia MJ, Gillespie CC, Kachur E, Schwartz MD, Ockene $\mathrm{J}$, Chepaitis AE, et al. Effect of communications training on medical student performance. JAMA 2003; 290(9):1157-65.

18. Egnew TR, Mauksch LB, Greer T, Farber SJ. Integrating communication training into a required family medicine clerkship. Acad Med 2004; 79(8):737-43.

19. Association of American Medical Colleges. Functions and Structure of a Medical School: Standards for Accreditation of Medical Education Programs Leading to the MD Degree. Washington, DC: Association of American Medical Colleges/Liaison Committee on Medical Education; 1997.

20. Evans BJ, Kiellerup FD, Stanley RO, Burrows GD, Sweet B. A communication skills programme for increasing patients' satisfaction with general practice consultations. Br J Med Psychol 1987; 60:373-8.

21. Roter DL, Hall J. Improving psychossocial address in primary care: Is it possible and what difference does it make? Toronto: International Conference on Doctor-Patient Communication;1991.

22. Brasil. Ministério da Educação. Conselho Nacional de Educação. Resolução CNE/CES № 4, de 7 de novembro de 2001. Institui Diretrizes Curriculares Nacionais do Curso de Graduação em Medicina. Disponível em: http:/ / www.mec.gov. br/cne/pdf/CES04.pdf. [Acesso em: 12 de Maio de 2007].

23. Brasil. Comissão Nacional de Residência Médica. Resolução CNRM № 008/2004, de 05 de agosto de 2004. Dispõe sobre o processo de seleção pública dos candidatos aos Programas de Residência Médica.Diário oficial da União 2004; 13 ago. Seção 1, p. 25.

24. Stoll C. O ensino-aprendizagem da relação médico-paciente: estudo de caso com estudantes da última fase do curso de medicina da Universidade Federal de Santa Catarina. 
[monografia] Florianópolis, Universidade Federal de Santa Catarina, 2005.

25. Patrício ZM, Casagrande JL, Araújo MF. Qualidade de vida do trabalhador: uma abordagem qualitativa do ser humano através de novos paradigmas. Florianópolis: Editora do autor; 1999.

26. R. Rossi SR, Batista NA. O ensino da comunicação na graduação em Medicina - uma abordagem. Interface - Comunic., Saúde, Educ. Educ. 2006; 10(19):93-102.

27. Smith CS, Irby DM. The roles of experience and reflection in ambulatory care education. Acad Med 1997; 72(1):32-5.

28. Roter DL, Stewart M, Putnam SM, Lipkin M, Jr., Stiles W, Inui TS. Communication patterns of primary care physicians. JAMA 1997; 277(4):350-6.

29. Haq C, Steele DJ, Marchand L, Seibert C, Brody D. Integrating the art and science of medical practice: innovations in teaching medical communication skills. Fam Med 2004;36 (Supl. S):43-50.

30. Mangione S, Kane GC, Caruso JW, Gonnella JS, Nasca TJ, Hojat M. Assessment of empathy in different years of internal medicine training. Med Teach 2002; 24(4):370-3.

31. Makoul G, Schofield T. Communication teaching and assessment in medical education: an international consensus statement. Netherlands Institute of Primary Health Care. Patient Educ Couns 1999;37(2):191-5.

32. Pascoe JM, Babbott D, Pye KL, Rabinowitz HK, Veit KJ, Wood DL. The UME-21 project: connecting medical education and medical practice. Fam Med 2004;36 (Supl. S):12-4.

33. Deveugele M, Derese A, De Maesschalck S, Willems S, Van Driel M, De Maeseneer J. Teaching communication skills to medical students, a challenge in the curriculum? Patient Education and Counseling. 2005; 58(3): 265-270

34. Ferreira FC, Silva RF, Aguer CB. Formação do Profissional Médico: a Aprendizagem na Atenção Básica de Saúde. Rev Bras. Educ. Med. 2007; 31(1):52-59.

35. Buyck D, Lang F. Teaching medical communication skills: a call for greater uniformity. Fam Med 2002; 34(5):337-43.

36. Novack DH, Volk G, Drossman DA, Lipkin M, Jr. Medical interviewing and interpersonal skills teaching in US medical schools. Progress problems, and promise. JAMA 1993; 269(16):2101-2105.

37. Makoul G. Essential elements of communication in medical encounters: the Kalamazoo consensus statement. Acad Med 2001;76(4):390-3.

38. Makoul G, Curry RH. The Value of Assessing and Addressing Communication Skills. JAMA 2007; 298:1057-1059.

39. Makoul G, Zick AMA; Green M. An Evidence-Based Perspective on Greetings in Medical Encounters. JAMA 2007; 298:1057-1059.

\section{CONFLITOS DE INTERESSE}

Declarou não haver.

\section{ENDEREÇO PARA CORRESPONDÊNCIA}

Desembargador Arno Hoeschel, 121 — apt. 802

Centro - Florianópolis

CEP.88015-620 / SC

E-mail:suelyg@terra.com.br 